\section{From fix to fit into the autoptic human brains}

\author{
Beatrice Paradiso, ${ }^{1,2,3}$ \\ Michele Simonato, ${ }^{3,4}$ Gaetano Thiene, ${ }^{1}$ \\ Anna M. Lavezzi ${ }^{2}$ \\ ${ }^{1}$ Cardiovascular Pathology Unit, \\ Department of Cardiac, Thoracic and \\ Vascular Sciences, University of Padua \\ Medical School \\ 2"Lino Rossi" Research Center for the \\ study and prevention of unexpected \\ perinatal death and SIDS Department of \\ Biomedical, Surgical and Dental \\ Sciences, University of Milan \\ ${ }^{3}$ Department of Medical Sciences, \\ Section of Pharmacology and National \\ Institute of Neuroscience, University of \\ Ferrara \\ ${ }^{4}$ School of Medicine, University Vita- \\ Salute San Raffaele, Milan, Italy
}

\begin{abstract}
Formalin-fixed, paraffin embedded (FFPE) human brain tissues are very often stored in formalin for long time. Formalin fixation reduces immunostaining, and the DNA/RNA extraction from FFPE brain tissue becomes suboptimal. At present, there are different protocols of fixation and several procedures and kits to extract DNA/RNA from paraffin embedding tissue, but a gold standard protocol remains distant. In this study, we analysed four types of fixation systems and compared histo- and immunostaining. Based on our results, we propose a modified method of combined fixation in formalin and formic acid for the autoptic adult brain to obtain easy, fast, safe and efficient immunolabelling of long-stored FFPE tissue. In particular, we have achieved an improved preservation of cellular morphology and obtained success in post-mortem immunostaining for NeuN. This nuclear antigen is an important marker for mapping neurons, for example, to evaluate the histopathology of temporal lobe epilepsy or to draw the topography of cardiorespiratory brainstem nuclei in sudden infant death syndrome (SIDS). However, NeuN staining is frequently faint or lost in post-mortem human brain tissues. In addition, we attained Fluoro Jade $\mathrm{C}$ staining, a marker of neurodegeneration, and immunofluorescent staining for stem cell antigens in the postnatal human brain, utilizing custom fit fixation procedures.
\end{abstract}

\section{Introduction}

It is often difficult to obtain reproducible immunohistochemical signals and to perform genomic analysis from postmortem brain tissues, because the elapsed time from the death of the patient, the time of fixation, and the time of embedding may influence the preservation of antigens and nucleic acids. ${ }^{1-5}$ In fact, researchers frequently prefer to exclude autoptic brain tissues from their experimental cases because the quality of nucleic acids varies in different post-mortem samples. However, it would be very interesting to obtain good quality autoptic tissue, both to have autoptic "normal" controls and to compare the genomic data from surgical and pathological specimens versus autoptic brain tissues.

The effect of post-mortem delay and fixation time are crucial preanalytical variables that can influence genomic analysis and immunohistochemistry outcome. A delay on fixation, as well as the time of formalin fixation can reduce the immunostaining efficiency. ${ }^{1,6}$ Preservation of proteins in relation to the post-mortem delay is sporadically calculated, and, in the routine setting, the post-mortem delay might range from a few hours to several days. Several biochemical changes occur in the cadaver, including a decrease in the $\mathrm{pH}$, which influences the exposure of target proteins and epitopes. However, this detection loss has received little attention from the scientific community. ${ }^{7,8}$ The usual fixative is $10 \%$ formalin, which consists $3.7 \%$ formaldehyde in water with $1 \%$ methanol. Formaldehyde is a reactive electrophilic substance; it reacts promptly with various functional groups of biological macromolecules in a cross-linking mode. ${ }^{9}$ Formalin degrades DNA and masks protein epitopes by forming covalent bonds between neighbouring proteins or nucleic acids. The initial cross-linking is formed by 24 to $48 \mathrm{~h}$ after penetration while the concluding may take about 30 days to generate the stable covalent cross linkages. The initial phase of the reaction is reversible while the later reaction becomes irreversible when there is high number of covalent bonds produced. This modifies the physicochemical state of tissue such as redox and membrane potentials of the tissue, changing surface charges and therefore alters the reactivity of cellular components. The reversible nature of the initial phase reaction is the basis of antigen retrieval in molecular techniques. To unmask epitopes for antibody binding in formalin-fixed tissue, several enzymatic and heat-induced antigen retrieval methods have been introduced. These methods are not completely successful for all antibodies and not all unmasking procedures are usable for every
Correspondence: Beatrice Paradiso, Cardiovascular Pathology Unit, Department of Cardiac, Thoracic and Vascular Sciences, University of Padua Medical School

Regional Registry for Cardio-CerebroVascular Pathology, Via A. Gabelli, 61, 35121 Padova, Italy.

Tel. +39.049.8272282 - Fax +39.049.8272294.

E-mail: beatrice.paradiso@unipd.it

Key words: Formalin fixation; formic acid; glacial acetic acid; ethanol; post-mortem brain tissue; NeuN; Fluoro Jade C; neural stem cell immunofluorescence staining.

Acknowledgments: We thank Dr. Fulvio Chiesa, Customer Application Specialist by Roche (Italy), Dr. Paolo Roncon, Department of Medical Sciences, Section of Pharmacology and Neuroscience Center, University of Ferrara, Italy, for their technical support, Dr. Graziella Alfonsi, "Lino Rossi" Research Center, Department of Biomedical, Surgical and Dental Sciences, University of Milan (Italy).

This study was supported by the Registry of Cardio-Cerebro-Vascular Pathology, Veneto Region, Italy.

Conflict of interest: The authors received no financial support for the research, authorship, and/or publication of this article.

Received for publication: 21 May 2018 Accepted for publication: 10 August 2018.

This work is licensed under a Creative Commons Attribution-NonCommercial 4.0 International License (CC BY-NC 4.0).

CCopyright B. Paradiso et al., 2018

Licensee PAGEPress, Italy

European Journal of Histochemistry 2018; 62:2944 doi:10.4081/ejh.2018.2944

antibody. ${ }^{9-14}$ Formic acid is generally used to virtually eliminate the risk of handling infectious material of autoptic tissues from patients with prionic diseases..$^{15}$ Formic acid pre-treatment may also produce an antigen retrieval effect, enhancing immunostaining. ${ }^{16-17}$

The present study aims at examining the effect of four different types of fixation on the morphological preservation, histostaining and immunoreactivities of several antibodies, against formalin-sensitive antigens, that do not produce optimal result in postmortem human brain tissue. In other words, we intend to compare formalin fixation, i.e. the standard and more common method, versus the traditional fixative solution mixed with formic acid and/or acid acetic respectively. We chose formic acid for its anti-prionic property and putative unmasking capacity. At the same time, we selected acetic acid, an efficient dehydrating agent, 
to reduce oedema of cerebral tissues. Clarke (1851) was the inventor of using a combination of alcohol and acetic acid for fixation, yet many authors attribute Carnoy (1887). ${ }^{18,19}$ Currently, the formalin, alcohol and acetic acid mixed protocol is utilized for foetal brain fixation. ${ }^{20,21}$ We decided to modify and utilize the foetal protocol for perinatal and postnatal brains, dividing the time of fixation in steps of four days for each passage (Table 1). We adapted protocols for adult brain fixation according to this postnatal protocol, to unify all of protocols in time point of multiple of four days. We chose the final time of formalin fixation in twenty days, according to the time course for both generation of stable covalent cross linkages, global volume shrinkage and formalin-induced changes of myelin. , $^{9,22}, 23 \mathrm{We}$ cut every fresh human brain in coronal macroslices of $1 \mathrm{~cm}$ of thickness to have fast penetration and fixation of the tissue. ${ }^{9}$ Every macroslice for each brain has been treated with different protocols to be tested, so, each brain has been fixed by all of our different protocols. The standard formalin fixation has been considered our internal negative control, because from this tissue we have never obtained positive immunostaining against formalin-sensitive antigens, as it usually happens with post-mortem brain tissues.

We propose a protocol to standardize the NeuN staining of autoptic brains by automatic machine staining. Furthermore, we propose to use Fluoro Jade C, a histochemical technique currently used only to detect neurodegeneration in animal models, for human autoptic diagnoses..$^{24,25}$

\section{Materials and Methods}

\section{Human adult brain tissue}

The study was approved by the "S. Anna" University Hospital Ethical Committee within the Project for Human White Matter Study of the Ferrara's University. ${ }^{26}$ post-mortem cerebral tissue was obtained from five adult patients without any previous history or symptoms of neurological disease. To over cross ethical problem related to the precocious extraction of the brains, and to reduce the variability of preanalytical phase, all tissues were extracted $40 \mathrm{~h}$ post-mortem ${ }^{8}$ During autopsy, the fresh brain was cut in coronal macro-sections of $1 \mathrm{~cm}$ thickness. In this way, four serial sections included the hippocampi. ${ }^{27}$ Following post-mortem macro-dissection, adjacent coronal slices were immersed in four different containers according to four different protocols of fixation (four containers for each post-mortem brain). One addi- tional coronal macro-section at the level of the hippocampus was obtained from a patient who died for a non-neurological cause and was affected by monolateral temporal post-traumatic epilepsy. This macrosection was fixed in $10 \%$ neutral-buffered formalin for almost three months.

All tissue blocks used in this study were from the temporal lobe and the hippocampus. To exclude any variability of antigen expression related to the Brodmann areas considered, we preliminary prepared different cortex blocks, taken from all cortical lobes of the brain fixed in formalin, therefore we compared antigens expression and nucleic acids extraction for each area.

\section{Human postnatal brain tissue}

Five autoptic postnatal brains were obtained from patients of about one month, who died for sudden unexpected infant death (SUID). We included a postnatal brain from a hypoxic ischemic encephalopathy case as our positive control. Again, brain was extracted $40 \mathrm{~h}$ post-mortem.

\section{Types of fixation}

We used four different methods of fixation for the adult human brain, which we named A, B, C and D type. In the A protocol, i.e. the traditional protocol of postmortem tissue fixation, we put macro-slices in 10\% neutral-buffered formalin for 21 days. In the B protocol, we used 10\% neutral-buffered formalin to fix but, at the $9^{\text {th }}$ day, we put the macro-slices in a $98 \%$ formic acid solution for 1 hour and then again in formalin. At the $21^{\text {st }}$ day, after the final dissection of the tissue blocks, we repeated the incubation in $98 \%$ formic acid for $1 \mathrm{~h}$, just before processing and paraffin embedding. In both the $\mathrm{A}$ and $\mathrm{B}$ protocols we changed the formalin after $24 \mathrm{~h}$ and then every four days, for a total of five times.

In the $\mathrm{C}$ protocol we used a liquid fixative (LF) based on a 10x glacial acetic acid solution in $10 \%$ neutral-buffered formalin. The LF was changed after $24 \mathrm{~h}$ and then every four days, for a total of four times. In the last four days we moved the slices in $70 \%$ ethanol. The D protocol was identical to the $C$, except at the $9^{\text {th }}$ and $17^{\text {th }}$ day we incubated slices in formic acid for $1 \mathrm{~h}$, just before the last passage in ethanol for four days (Table 1).

The macro-slice from the epileptic post-traumatic brain was fixed using the A protocol for three months. For postnatal brains, we used the LF described above for protocol $\mathrm{C}$, which was changed every day for 8 days. Thereafter, brains were plunged into $70 \%$ ethanol for 4 days, then in fresh $80 \%$ ethanol for another 4 days and finally cut in macro-slices to conclude the fixation using a $95 \%$ ethanol solution for 4 days (Table 2).

\section{Paraffin embedding}

To exclude the variability due to postfixation processing, all tissue blocks were processed and embedded in paraffin within 1 week. Paraffin embedded blocks were cut in $7 \mu \mathrm{m}$ sections for histology and immunohistochemistry, or in $10 \mu \mathrm{m}$ sections for genomic analysis, using a microtome (Leica, Wetzlar, Germany). Sections were mounted on adhesive microscopy slides (Superfrost plus, Thermo Scientific, Waltham, MA, USA) and assigned for staining and RNA purification and amplification to researchers whot were blind of the group to which each section belonged.

Table 1. Adult fixation protocols.

\begin{tabular}{ll}
\hline A & 1 day $+5 \times 4$ days in formaline (21 days) \\
B & $\begin{array}{l}1 \text { day }+2 \times 4 \text { days ( } 9 \text { days) in formaline }+ \text { formic acid } 1 \mathrm{~h}+3 \times 4 \text { days (12 days) in formaline } \\
+ \text { formic acid } 1 \mathrm{~h} \text { (before processing) }\end{array}$ \\
\hline C & 1 day $+4 \times 4$ days (17 days) in $\mathrm{LF}$ (Formaline + acetic acid) + EtOH $70 \mathrm{X} 4$ days \\
D & 1 day $+2 \times 4$ days $(9$ days) in $\mathrm{LF}+$ formic acid $1 \mathrm{~h}+2 \times 4$ days in $\mathrm{LF}+$ formic acid $1 \mathrm{~h}$ \\
& + EtOH $70 \mathrm{X} 4$ days
\end{tabular}

Table 2. Perinatal fixation protocol.

\begin{tabular}{lccc} 
Liquid fixative* & EtOH 70X & EtOH $80 \mathrm{X}$ & $\begin{array}{c}\text { Cutting in } \\
\text { macroslices }\end{array}$ \\
$\begin{array}{l}8 \text { days in liquid fixative } \\
4 \text { days } \\
\text { (changed each day) }\end{array}$ & 4 days & 4 days & \\
\hline
\end{tabular}

*Glacial acetic acid (10x solution) in 10\% neutral-buffered formalin 


\section{Histology and immunohistochem- istry}

\section{Automatized staining and immunostaining}

The haematoxylin and eosin (H\&E) stain was automatically performed using Leica Biosystems for two sections of each block. For immunostaining, we used an automatic and clinically validated instrument (Ventana Benchmark Ultra Systems from Roche Tissue Diagnostics). For all antibodies, we used the automatic $\mathrm{CC} 1$ Ventana pretreatment (Cell Conditioning Solution, Ventana Catalog Number 950124) for $52 \mathrm{~min}$. We used two Ventana prediluted custom-fit antibodies, the antiNeurofilament antibody (clone 2F11, mouse monoclonal, Roche Number 05267714001, by Cell Marque CMC26610021) and the anti-Glial Fibrillary Acidic Protein antibody (GFAP, clone EP672Y, rabbit monoclonal, Roche Number 05269784001, by Cell Marque CMC43450021). For NeuN, neuronal nuclear antigen, we chose the anti NeuN/Fox3 antibody (clone N/A60, mouse monoclonal, by Immunological Sciences MAB-90228; 1:100). All three antibodies were incubated for $1 \mathrm{~h}$. The UltraView DAB procedure (Benchmark Ultra System) was used for detection.

\section{Manual NeuN and Nestin immunostaining}

Manual immunohistochemistry was performed as previously described on the long-term fixation slides from the epileptic post-traumatic brain. ${ }^{28,29}$ Sections were dewaxed (2 washes in xylol for $10 \mathrm{~min}, 5$ min in ethanol $100 \%, 5 \mathrm{~min}$ in ethanol $95 \%$ ) and then rehydrated in distilled water for 5 minutes and in PBS $1 \mathrm{x}$ for $10 \mathrm{~min}$. The neuronal nuclear antigen was unmasked using a commercially available kit (Unmasker, Diapath), according to the manufacturer's instructions. We then employed the Dako Cytomation EnVision ${ }^{\circledR}+$ Dual Link System-HRP (DAB+) kit. After washing in PBS $1 x$, sections were incubated at room temperature for $10 \mathrm{~min}$ with Endogenous Enzyme Block, to quench endogenous peroxydase activity, and then incubated with the primary antibodies. For NeuN, we used the anti NeuN/Fox3 antibody (clone N/A60, mouse monoclonal, by Immunological Sciences MAB-90228; from 1:25 to 1:200). We used anti-nestin (mouse monoclonal, Immunological Sciences MAB-10430; $1: 20)$, without unmasking, in the postnatal brain. For NeuN, we performed an overnight incubation at $4^{\circ} \mathrm{C}$ in humid atmosphere; for nestin, 100 minutes at room temperature in humid atmosphere. Slices were then rinsed twice in PBS $1 \mathrm{x}$ and incubated for another 30 min with Labeled PolymerHRP [Dako Cytomation EnVision ${ }^{\circledR}+$ Dual
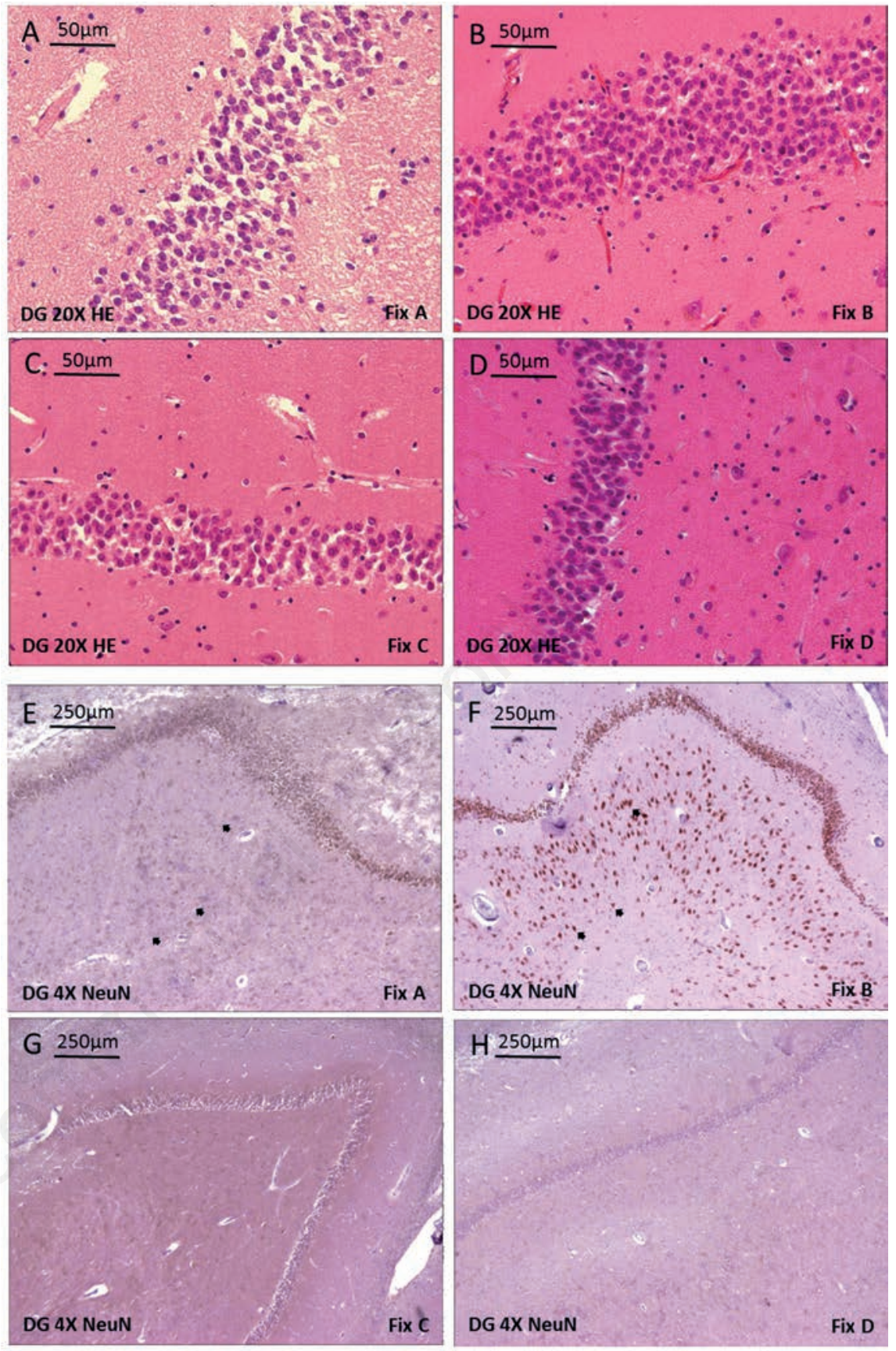

Figure 1. Human dentate gyrus in post-mortem cases. Hematoxylin and eosin staining (HE), magnification 20x and automatized NeuN immunohistochemistry performed in four different fixation protocols obtained from the same autoptic case, magnification $4 x$. A) $\mathrm{HE}$ in formalin fixation (classic protocol); B) $\mathrm{HE}$ in formalin fixation and formic acid treatment; C) $\mathrm{HE}$ in liquid fixative (LF) based on glacial acetic acid and formalin solution; D) HE in LF and formic acid treatment; E) NeuN staining in A protocol; F) NeuN staining in B protocol; G) NeuN staining in C protocol; $\mathrm{H}$ ) NeuN staining in D protocol. HE staining shows that by $B$ protocol the tissue matrix is more compact, without empty and irregular artefactual spaces, nuclei and nucleoli are more visible and better preserved and cell shape appears well represented (B). Automatized staining for NeuN results surely positive with little background exclusively in B protocol (F). Automatized NeuN immunostaining in fixation A protocol shows high level of background and paler staining for CA4 neurons (black arrow heads) and more loose parenchyma (E), compared to automatized NeuN staining in B protocol (F). 
Link System-HRP (DAB+)]. The reaction product was detected as a brown substrate using 3,3-diamino-benzidinetetrahydrochloride (DAB). Finally, sections were mounted using a water-based mounting medium (Shur Mount ${ }^{\mathrm{TM}}$, TBS). The specificity of immunolabeling was verified with a control in which the primary antibody was omitted.

\section{Fluoro-Jade C (FJC) staining}

Sections were dewaxed as described above and incubated in a solution containing $1 \% \mathrm{NaOH}$ in $80 \%$ ethanol (5 min), then in $70 \%$ ethanol $(2 \mathrm{~min})$, then in distilled water ( $2 \mathrm{~min})$. They were then incubated for 10 minutes in $0.06 \%$ potassium permanganate, washed for 2 minutes in distilled water, and transferred to a $0.001 \%$ FJC staining solution (IS-0012, FJC solution 1000x, Immunological Sciences). After staining, sections were rinsed in distilled water, counterstained with $0.0001 \%$ 4',6-diamidino-2-phenylindole (DAPI) for $15 \mathrm{~min}$, washed again, and air dried.

\section{Manual immunofluorescence}

Sections were dewaxed, rehydrated, and unmasked as described above. After washing in PBS, sections were incubated with Triton X-100 (0.3\% in PBS $1 \mathrm{x}$, room temperature, $10 \mathrm{~min}$ ), washed twice in PBS $1 \mathrm{x}$ and incubated with 5\% BSA and $5 \%$ serum of the species in which the secondary antibody was produced, for $30 \mathrm{~min}$. They were then incubated with the primary antibodies overnight at $4{ }^{\circ} \mathrm{C}$, as follows: nestin (mouse monoclonal, Chemicon) and SOX2 (rabbit polyclonal, Immunological Sciences) 1:10 and 1:25 respectively. After 5-min rinses in PBS, sections were incubated with Triton X-100 (as above, 30 min), washed in PBS and incubated with the goat anti-mouse, Cy2 (Cyanine)-conjugated, secondary antibody (1:50 dilution; Jackson ImmunoResearch) for mouse primary antibodies, or with a goat anti-rabbit, Texas red-conjugated, secondary antibody (1:100; Jackson ImmunoResearch) for rabbit primary antibodies, at room temperature for $3.5 \mathrm{~h}$. After staining, sections were washed in PBS, counterstained with $0.0001 \%$ DAPI for $15 \mathrm{~min}$, and washed again. Coverslips were mounted using water-based anti-fading Gel/Mount (Biomeda).

\section{DNA/RNA extraction}

Total DNA/RNA were extracted using a total nucleic acid purification kit (RecoverAll Total Nucleic Acid Isolation Kit, Ambion Life Technologies, Foster City, CA, USA), from each paraffin embedded block by $10 \mu \mathrm{m}$ thickness slices. The concentration and the quality of nucleic acid were investigated using a
NanoDrop 1000 spectrophotometer (Thermo Scientific) and an Agilent 2100 Bioanalyzer. All of the procedures were produced according to our previously published protocols. ${ }^{30,31}$
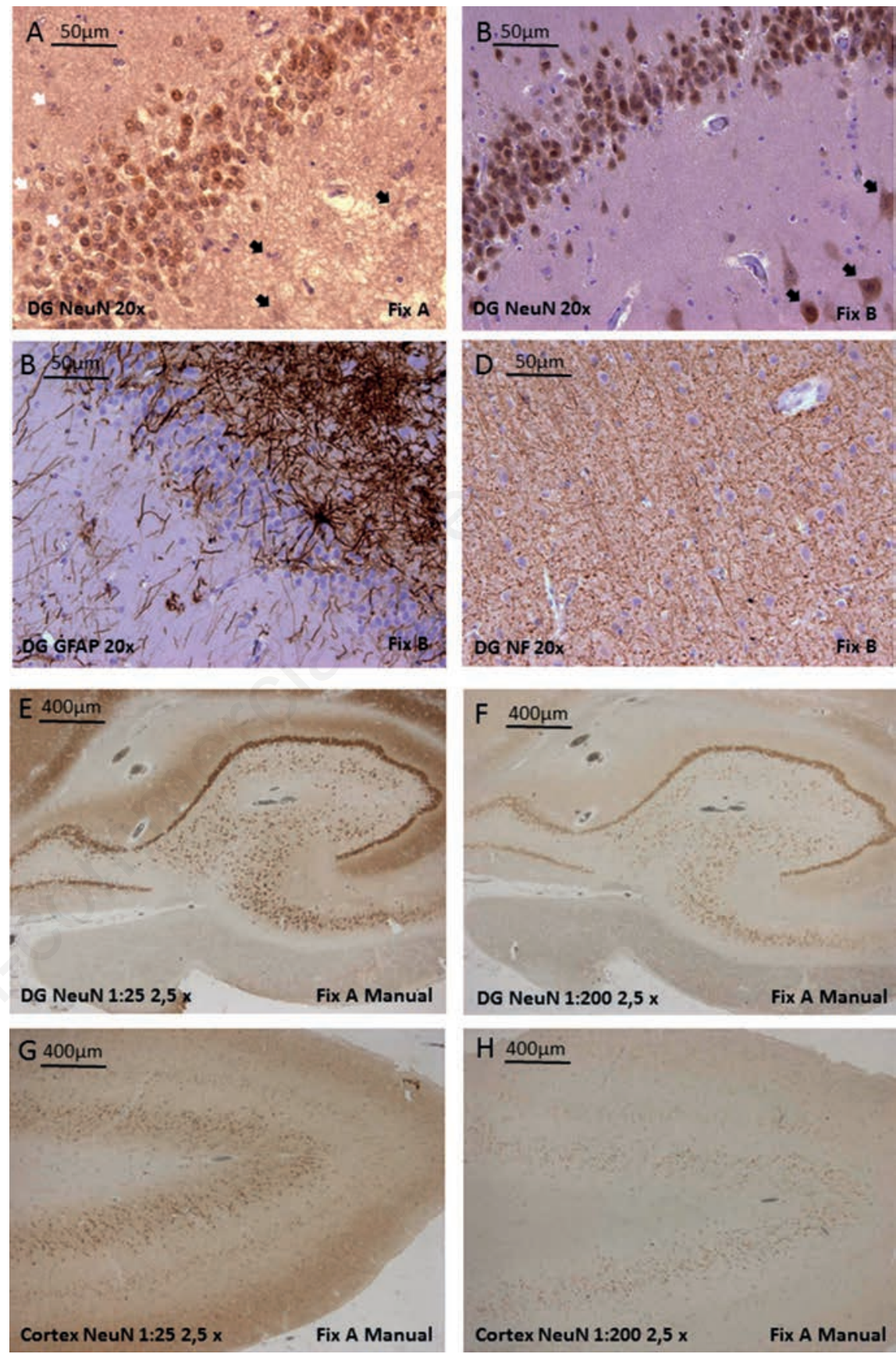

\section{Analysis}

Sections were analysed using a Leica microscope (DMRA2), by three expert pathologists under double blind conditions.

Cell shape, consistency of parenchymal
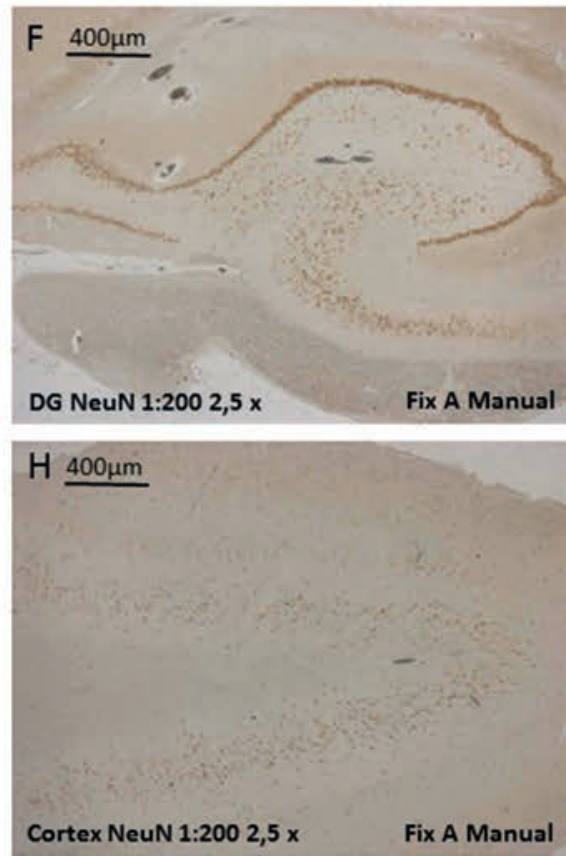

Figure 2. Human dentate gyrus in post-mortem cases. Automatized immunohistochemistry performed against NeuN, GFAP and NF brain antigen markers, magnification 20x (A-D) and manual staining against NeuN antigen, magnification $2.5 \mathrm{x}(\mathrm{E}-\mathrm{H})$. A) Automatized NeuN immunostaining in A-type fixation protocol appears paler with more background, false positivity for glia and astrocytes (white arrow heads) and negativity of CA4 area neurons (black arrow heads), compared with B. B) Automatized NeuN immunostaining in B-type fixation protocol. C) Automatized GFAP immunostaining in B-type fixation protocol. D) Automatized NF immunostaining in B-type fixation protocol. E-H) Human Hippocampus in long-term fixation of the same post-mortem case. Manual immunostaining in A-type fixation protocol, with different antibody concentration respectively in E,G) and F,H). 
matrix, nucleolar and nuclear preservation as well as immunohistochemistry positivity, have been evaluated using objectives from $5 \mathrm{x}$ to $20 \mathrm{x}$ by means a semiquantitative method based on score among 0 and +++ . The degree of neurodegeneration was analysed on Fluoro-Jade C sections, based on a modification of a previously described "neurodegeneration score" for animal models. ${ }^{32}$ The score range spanned from 0 (absence of Fluoro-Jade C-positive neurons) to +++ (maximal neurodegeneration pattern in the hippocampus). +++ was given with many positive cells with confluent fluorescence.

\section{Results}

We compared four different fixation protocols. Comparison was made between: A protocol (10\% neutral-buffered formalin for 21 days); B protocols (10\% neutralbuffered formalin for 21 days with two intervals of 1 hour each, at day 9 and 21, in which tissue was dipped in a $98 \%$ formic acid); C protocol (LF every four days and $70 \%$ ethanol for the last four days); D protocol (as $\mathrm{C}$ except at the day 9 and 17, when tissue was dipped in a $98 \%$ formic acid solution). We chose A protocol, traditionally utilized for most of the histochemical techniques, as our negative internal control to confirm and evaluate morphological observations obtained by others.

The automatically performed $\mathrm{H} \& \mathrm{E}$ staining was produced on the four differently fixed brain tissues, obtained from the same patient (Figure $1 \mathrm{~A}-\mathrm{D}$ ). We observed a better preservation of the cell shape, more compact parenchymal matrix and nucleolar and nuclear preservation with the formalinformic fixation (B protocol, Figure 1B). For the perinatal and postnatal brain, characterized by a high content of water, ${ }^{33}$ we utilized only one approach of fixation, slightly modifying the fixation procedure of $\mathrm{C}$ protocol ( $\mathrm{C}$ modified protocol). In fact, we used acetic acid addition to formalin with different ethanol steps, dehydrating agents. For immunostaining, we used an automatic and clinically validated procedure based on Ventana Benchmark Ultra systems from Roche Tissue Diagnostics. Both for NeuN and for easier to detect antibodies like GFAP, the quality of immunohistochemistry was superior by using the B protocol. Also the neurofilament and GFAP staining were better in B-type-tissues compared with the other fixations (Figures $1 \mathrm{E}-\mathrm{H}$ and $2 \mathrm{~A}, \mathrm{~B}$ ).

Manual immunohistochemistry for $\mathrm{NeuN}$ (Figure $2 \mathrm{C}-\mathrm{F}$ ) and nestin (Figure 4A) was performed on adult and postnatal cerebral tissues, respectively, by means a stronger and drastic manual unmasking pro- tocol. We were able to perform an excellent staining on macroslices fixed in classic formalin protocol (A protocol), for adult brain tissue, whereas we utilized the previously described foetal/ perinatal fixation protocol (C modified protocol), for the nestin immunostaining of postnatal brain tissue.

Regarding identification of the best antigen retrieval protocol, we verified different treatments: 1 . CC1 Ventana pre-treatment; 2 . microwave irradiation by means of a commercially available kit (Unmasker, Diapath); 3. formic acid pre-treatment; 4. Triton X-100 permeabilization; 5. a combination of 1 and 3 , or 2 and 4 .

We tested neurofilament (Figure 2C), GFAP (Figure 2D) and NeuN antigen expression in adult brains (Figures 1 E-H and 2 A,B,E-H) and Nestin and SOX2 in postnatal brain (Figures 4A and $5 \mathrm{~A}-\mathrm{E}$ ). For post-mortem adult brains, we applied
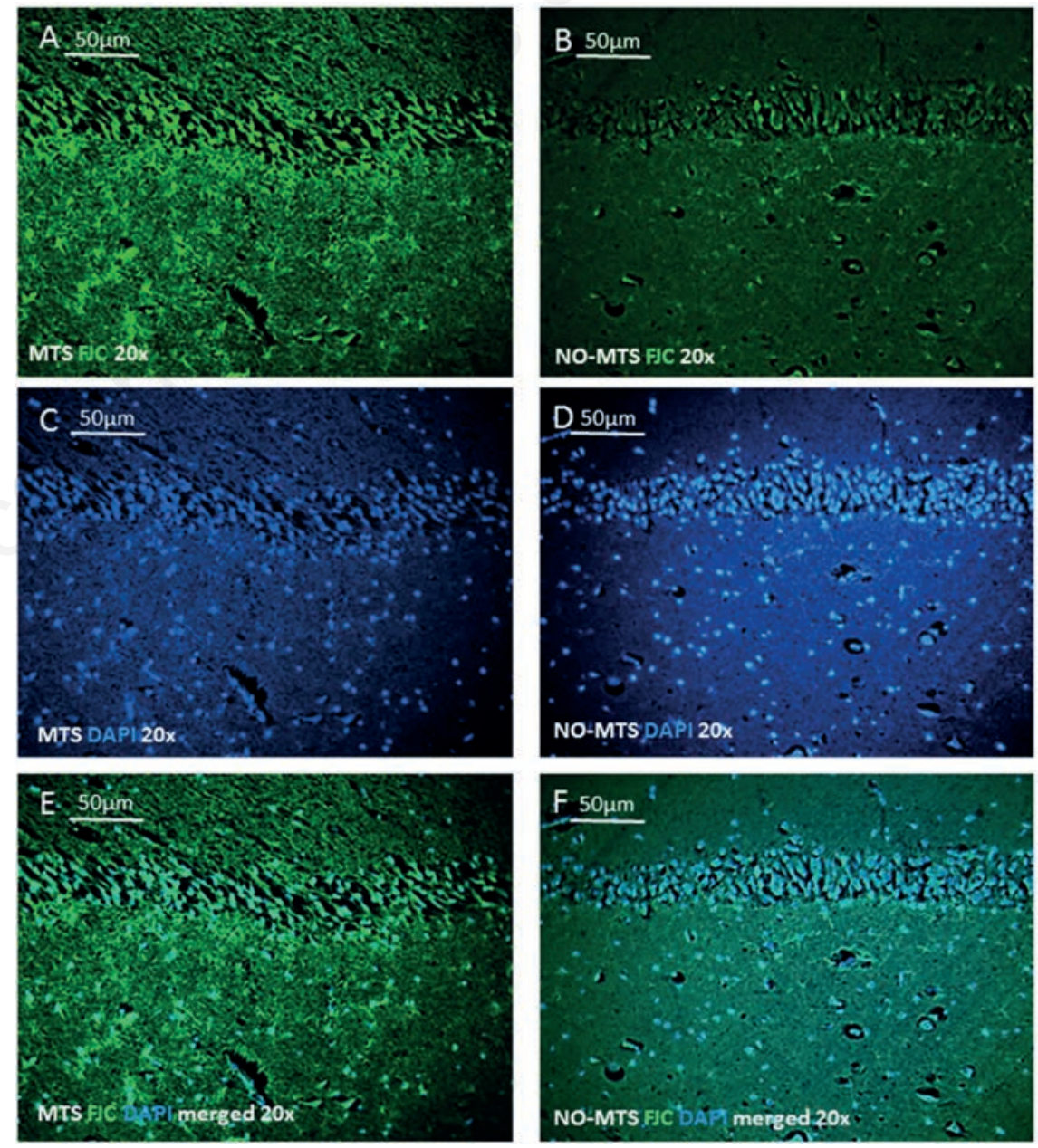

Figure 3. Human adult Fluoro Jade C (FJC) staining of hippocampal neurodegeneration, magnification 20x. A) Monolateral temporal post-traumatic epilepsy with left mesial temporal sclerosis (MTS), FJC in green; B) Controlateral temporal lobe in absence of neurodegeneration, FJC in green; C) DAPI in blue of left MTS; D) DAPI in blue of contralateral side. E, F) merged view of A-C and B-D. 
any problems. Score for FJC positivity was totally in advantage for temporal lesion in post-traumatic epilepsy and for postnatal brain with hypoxic ischemic encephalopathy on the not-lesioned contralateral hippocampus $(+++/+)$ and respectively on SUID cases $(+++/+)$. By means of this fixation method, concomitant with the immunofluorescence protocol, we detected neural stem cells positive for nestin and SOX2 (Figure 5 A-E).

Initial data of RNAs extraction and DNA amplification display apparently homogeneous and similar results with the four types of fixation (Table 3). Randomly we repeated the NeuN staining by automatized Ventana procedure, on different postmortem brain samples arrived to our attention for diagnostic purpose and obtained the same result.

\section{Discussion}

The study of brain histology and protein expression represents a fundamental step in the understanding of brain development and physiopathology of the Central Nervous System diseases. Protein expression in FFPE tissue has been analysed, however, despite advances in molecular technologies, the quality of RNA and protein from fixed paraffin-embedded tissue remains variable. In particular, in autoptic cases with longterm fixation and high variability deriving from the coroner post-mortem practices, both the immunohistochemistry and gene and RNA detection, request more accurate and detailed validation of custom fit procedures. ${ }^{7,8,34,35}$ Regarding to the preanalytical phase of post-mortem delay of autopsy, we choose the interval between death and autoptic procedures around 40 hours. We standardized this interim period on $40 \mathrm{~h}$, because it corresponds to the time of brain extraction that is considered adequate for the ethical problems (usually $24 \mathrm{~h}$ ) and in which we do not observe any alteration in the intensity of staining of the more common neuronal and glial antigens (between 33 and 48 h). ${ }^{8}$ Recently, Thom and coworkers have published a review, in which they provide a framework for best practices, integration of clinical, pathological and molecular genetic investigations in Sudden Unexpected Death in Epilepsy (SUDEP). ${ }^{36}$ Authors underlined the lack of sufficient numbers of suitable control cases and tissues and lack of standardized guidance documents for conducting autopsies. Our intent has been to follow these suggestions by evaluating different techniques of fixation, antigen retrieval, immunohistochemistry and/or fluo-staining and RNA extraction in formalin-fixed paraffin-embedded tissue
Table 3. RNA-DNA extraction.

\begin{tabular}{|c|c|c|c|c|c|}
\hline & & & 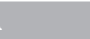 & & \\
\hline Fixations & $74 / 13$ & $15 / 13$ & D5/13 & $80 / 13$ & $81 / 13$ \\
\hline A & $* * * * * *$ & $* * *$ & $* / * *$ & & \\
\hline B & $* * * * * * *$ & & & & $* * *$ \\
\hline $\mathrm{C}$ & & & & $* / * *$ & \\
\hline D & $* * * *$ & & & $* *$ & $* * *$ \\
\hline
\end{tabular}

RNA ratio: *1.55-1.64; **1.65-1.74; ***1.75-1.84; **** 1.85-1.99; DNA ratio: *1.55-1.64; **1.65-1.74; ***1.75-1.80.
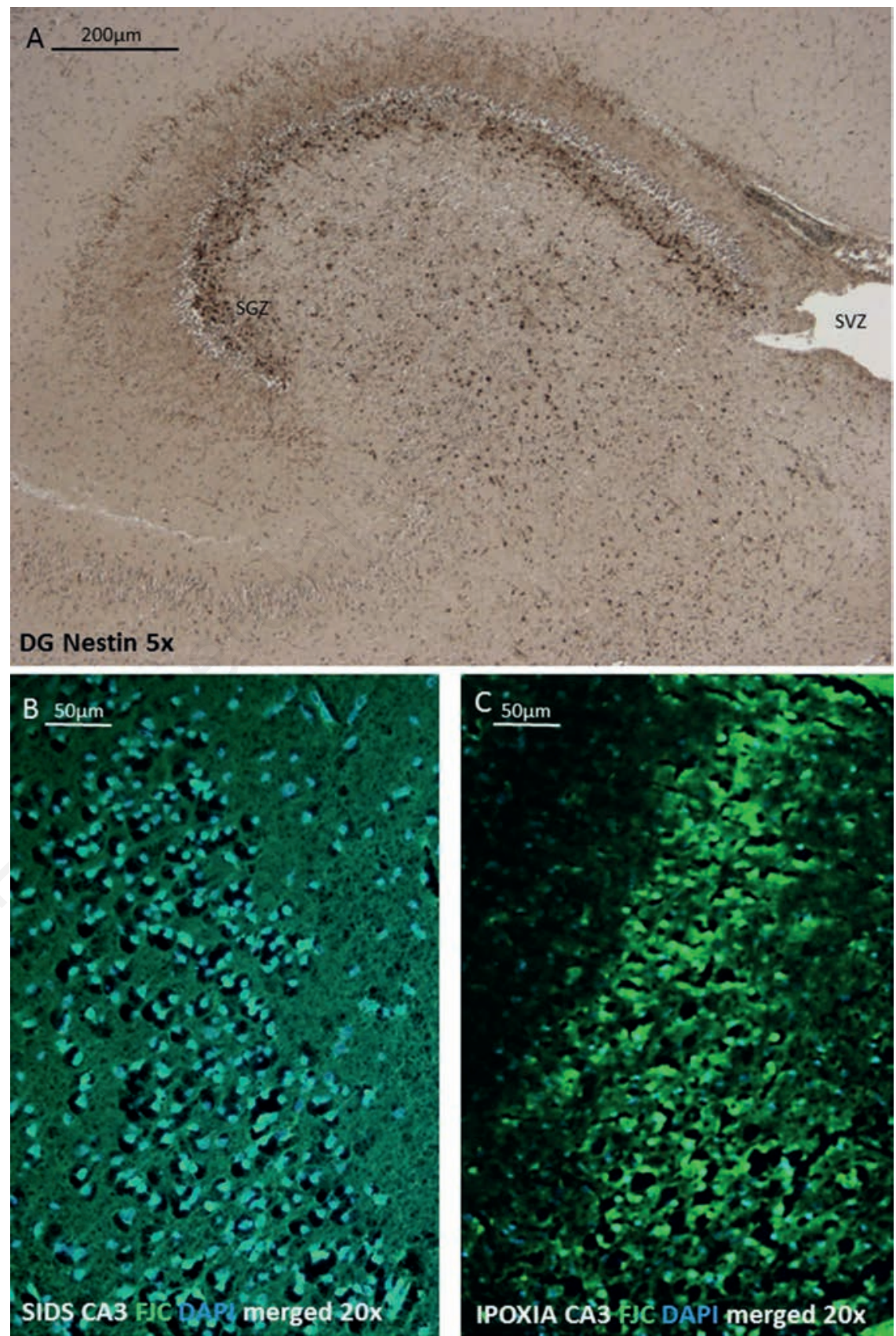

Figure 4. Human postnatal dentate gyrus in SIDS case. Nestin immunostaining, magnification 5x and Fluoro Jade $C$ (FJC) neurodegeneration detection, magnification 20x. A) Nestin immunostaining in LF protocol: nestin- positive neural precursors are migrating from the subgranular (SGZ) and subventricular zone (SVZ); B) Human postnatal FJC staining (in green) of CA3 area of hippocampus in SIDS case, without any aspect of neurodegeneration, compared with $\mathrm{CA} 3$ area of perinatal FJC positive hypoxic ischemic encephalopathy in the control case (C), nuclei stained in blue by DAPI. 
from post-mortem brains in long-term fixation.

It is a consolidated and firm data that long-term formalin fixation for postmortem human brain produces an impairment of immunolabeling with formalin-sensitive antibodies. ${ }^{1}$ Many expensive procedures have been yet tested without definitive good results. NeuN automatized immunostaining persists, for example, a prohibitive immunostaining for postmortem brain tissues, as you can see by traditional protocol of formalin fixed brain macroslices (A protocol), in which $\mathrm{NeuN}$ staining carries on negative (Figure 1E). In other words, we chose A protocol as our negative internal control. Therefore, we decided to compare different substances to try to improve the staining results for formalin-sensitive antibodies. We considered formic acid and glacial acetic acid because respectively are normally utilized as antiprionic and dehydrating agents. Therefore, to determine the best strategy to maintain the integrity of post-mortem brain tissue, cell morphology and nuclear structure, we compared four different fixation protocols.

Comparison was made between: A protocol, exclusively formalin; B protocols, formalin and formic acid solution; $\mathrm{C}$ protocol, procedure with foetal autopsy dedicated $\mathrm{LF}$, appropriate to dehydrate brain tissues and a mix method between $\mathrm{B}$ and $\mathrm{C}$ protocol (D protocol), to eventually test their synergism.

Preliminary data showed that the postmortem brain tissue in formalin fixed and formic acid treated (B-type protocol) lone preserved good morphology in H\&E. We scored histochemical results by microscope analysis of pathologists, however results were totally in favour of the B-type protocol. Tissue matrix is more compact, without empty and irregular artefactual spaces, nuclei and nucleoli are more visible and better preserved and cell shape appears well represented (Figure 1 A-D).

Regarding identification of the best antigen retrieval protocol, it is well known that formalin and paraffin embedding both abolish or drastically reduce immunoreactivity with the majority of the antibodies used routinely in neuropathological studies, especially in autoptic post-mortem tissues. We verified different treatments: 1 . CC1 Ventana pre-treatment; 2 . microwave irradiation by means of commercially available kit (Unmasker, Diapath); 3. Formic acid pre-treatment; 4. Triton X-100 permeabilization; 5 . a combination of 1 and 3 , or 2 and 4 .

Our study evidenced that, for adult post-mortem brain tissue, the B protocol is the preferable approach for the automatized antigen detection, while $\mathrm{A}$ protocol, i.e. exclusively formalin, resulted the best fixation for manual immunostaining only when it is associated with a particular strong procedure of unmasking by microwave irradiation and Unmasker Diapath solution. Excellent automized NeuN staining (Figures 1 E-H and $2 \mathrm{~A}, \mathrm{~B}$ ) appears true pos-
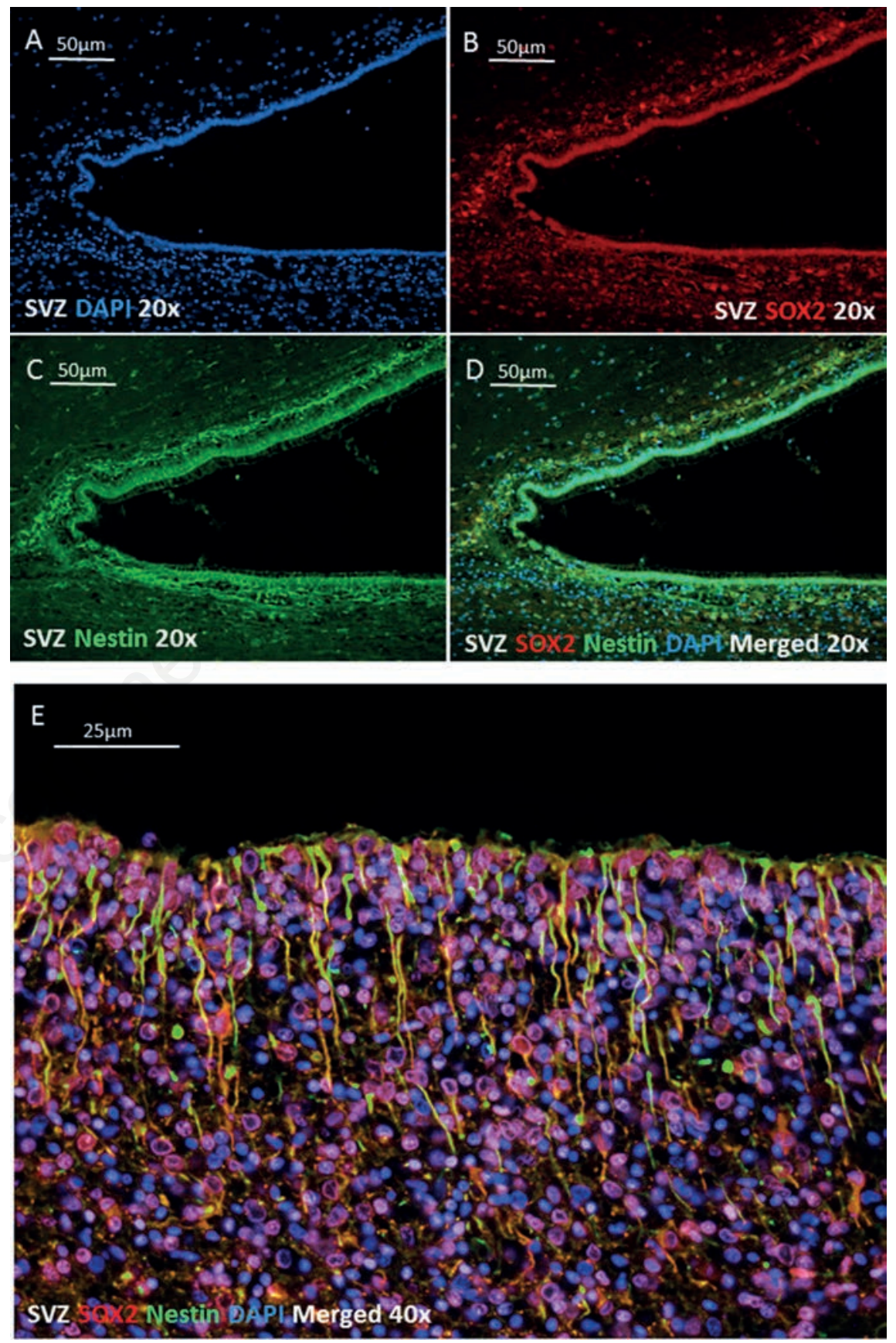

Figure 5. Human postnatal neurogenesis in subventricular zone (SVZ) by antigen of stemness in SIDS case. Immunofluorescence staining in LF fixation protocol, magnification 20x. A) Nuclei in blue, DAPI; B) SOX2 in red; C) Nestin in green; D) merged image of A, B, C; E) Human postnatal neurogenesis in subventricular zone (SVZ) in SIDS case. Merged image of immunofluorescence staining: nuclei in blue, DAPI; nestin in green; SOX2 in red, in LF fixation protocol, magnification 40x. 
exclusively by $\mathrm{B}$ fixation (black arrow heads in Figures 1 E,F and 2 A,B). In addition, GFAP and the neurofilament staining were better in B-type-tissues compared with the other fixations too (Figure 2 C,D). We consider our automatized $\mathrm{B}$ protocol an interesting method to quantify clinical and research data from post-mortem tissue and a proficient approach to standardize NeuN detection according to shared validation system in different Institutions. ${ }^{36}$ Obviously, this approach has to be tested on long-fixed brains from brain bank collections. On the other hand, the manual protocol of immunostaining allows to control the development of the staining during the DAB exposure (checking under microscope) and reduce the problem of background (Figures 2 E-H). For the same reason the use of manual colorimetric staining could be suggested for paediatrics cases. Nevertheless, manual NeuN staining could be the first choice for dysplasia or brain cortical malformation and dysmorphisms in perinatal deaths, an optimal approach to study the hippocampal sclerosis and granule cell pathology in postmortem epileptic cases and SUDEP. The manual technique, more accurate than the automatic one, could be necessary to distinguish hypoplasia or dysgenesia of cardiorespiratory nuclei implicated in SIDS. For the first time in the Literature, we demonstrated an optimal fixation with LF (C protocol) of postnatal brain. This type of protocol, in general, is utilized for embryonic and foetal brains because it has a great power of dehydration. ${ }^{37}$ In fact, according to Widdowson and Dickerson, the postnatal brain maintains a similar water content as foetus of 20-22 gestational weeks. ${ }^{38}$

The striking decrease in brain water in adult aging resembles the decrease in brain water observed in early life through adulthood: $20-22$ week foetus $92 \%$, new-born $90 \%$ and adult $77 \%$. By combined approach of double antigen retrieval in immunofluorescent staining, we distinguished nestin and SOX2 antigens of stemness in neurogenetic area of postnatal brain, confirmed by colorimetric approach (Figure 4A).

There are very few data in the Literature regarding the development and application of novel histochemical tracer of neurodegeneration in human brain, for this reason we tested Fluoro-Jade C, usually utilized in animal models of neurodegeneration. According our result, we propose FluoroJade $\mathrm{C}$ staining in adult and babies autopsies (Figure $3 \mathrm{~A}-\mathrm{F}$ ) as an easy and fast histofluostaining to suppose the presence of neurodegeneration cells in such amount as to exclude the sudden death. ${ }^{24,32}$

Moreover, nucleic acid information does not seem lost through these four fixation techniques. Our current data of RNAs extraction show apparently homogeneous and similar results for the four types of fixation protocol and these outcomes are very interesting especially because it is possible utilize LF and ethanol for perinatal brain tissue without lose the genomic information of cerebral samples. In fact, has to be considered mandatory for SIDS cases to realize the genomic analysis of possible chanellopathies or potential eredopathies in infant sudden death. Furthermore, by our LF fixation protocol for postnatal brain tissue we were able to detect infective diseases utilizing Altona Diagnostics assay in efficient way (data not shown).

The processing parameters that determine the quality of RNAs and proteins in fixed paraffin-embedded tissues have been traditionally recognized. There are four important factors that are involved in RNA and protein preservation: time before fixation, methods of fixations, length of fixation and exclusively for protein detection, antigen retrieval techniques. The results of the different methods of fixation confirm that post-mortem brain tissue exhibited different affinity for antibodies or histo and/or fluostaining and it is possible to adjust different protocols according to molecular requirement or to the patient ages. It is advisable in the future to test the different protocols for very long-lasting fixations, such as those of tissue banks, and drafting of the procedural guidelines.

\section{References}

1. Liu JY, Martinian L, Thom M, Sisodiya SM. Immunolabeling recovery in archival, post-mortem, human brain tissue using modified antigen retrieval and the catalyzed signal amplification system. J Neurosci Methods 2010;190:4956.

2. Eisele S, Krumbholz M, Fischer MT, Mohan H, Junker A, Arzberger T, et al. Prospects of transcript profiling for mRNAs and MicroRNAs using formalin-fixed and paraffin-embedded dissected autoptic multiple sclerosis lesions. Brain Pathol 2012;22:607-18.

3. Carturan E, Tester DJ, Brost BC, Basso C, Thiene G, Ackerman MJ. postmortem genetic testing for conventional autopsy-negative sudden unexplained death: An evaluation of different DNA extraction protocols and the feasibility of mutational analysis from archival paraffin-embedded heart tissue. Am J Clin Pathol 2008;129:391-7.

4. Wimmer I, Tröscher AR, Brunner F, Rubino SJ, Bien CG, Weiner HL, et al. Systematic evaluation of RNA quality, microarray data reliability and pathway analysis in fresh, fresh frozen and formalin-fixed paraffin-embedded tissue samples. Sci Rep 2018;8:6351.

5. Marwitz S, Kolarova J, Reck M, Reinmuth N, Kugler C, Schädlich I, et al. The tissue is the issue: improved methylome analysis from paraffinembedded tissues by application of the HOPE technique. Lab Invest 2014;94: 927-33.

6. Sas L, Van Laere S, Dierick AM, Duwel V, De Pauw A, Van Den Eynden G, et al. Study assessing the quality of quantification of estrogen receptor protein expression by immunohistochemistry and gene expression in breast cancer. Pathol Res Int 2014;2014:372653.

7. Engel KB, Moore HM. Effects of preanalytical variables on the detection of proteins by immunohistochemistry in formalin-fixed, paraffin-embedded tissue. Arch Pathol Lab Med 2015;135: 537-43.

8. Lundström Y, Lundström P, Popova SN, Lindblom RPF, Alafuzoff I. Detection of changes in immunohistochemical stains caused by postmortem delay and fixation time. Appl Immunohistochem Mol Morphol 2018. doi: 10.1097/PAI.0000000000000658. [Epub ahead of print].

9. Thavarajah R, Mudimbaimannar VK, Elizabeth J, Rao UK, Ranganathan K. Chemical and physical basics of routine formaldehyde fixation. J Oral Maxillofac Pathol 2012;16:400-5.

10. Battifora H, Kopinski M. The influence of protease digestion and duration of fixation on the immunostaining of keratins. J Histochem Cytochem 1986;34:1095-100.

11. Shi SR, Key ME, Kalra KL. Antigen retrieval in formalin-fixed, paraffinembedded tissues: an enhancement method for immunohistochemical staining based on microwave oven heating of tissue sections. J Histochem Cytochem 1991;39:741-8.

12. Evers P, Uylings HBM. Effects of microwave pre-treatment on immunocytochemical staining of vibratome sections and tissue blocks of human cerebral cortex stored in formaldehyde fixative for long periods. J Neurosci Methods 1994;55:163-72.

13. Evers P, Uylings HBM. An optimal antigen retrieval method suitable for different antibodies on human brain tissue stored for several years in formaldehyde fixative. J Neurosci Methods 1997;72:197-07.

14. Hideaki K, Ryong-Woon S, Koichi O, Hiroyuki H, Shigeo M, Tetsuyuki K. Enhanced antigen retrieval of amyloid $\beta$ immunohistochemistry: Re-evaluation 
of amyloid $\beta$ pathology in Alzheimer disease and its mouse model. J Histochem Cytochem 2012;60: 61-9.

15. Brown P, Wolff A, Gajdusek DC. A simple and effective method for inactivating virus infectivity in formalin-fixed tissue samples from patients with Creutzfeldt-Jakob disease. Neurology 1990;40:887-90.

16. Liu JY, Martinian L, Thom M, Sisodiya SM. Immunolabeling recovery in archival, post-mortem, human brain tissue using modified antigen retrieval and the catalyzed signal amplification system. J Neurosci Methods 2010;190:49-56.

17. Pikkarainen M, Martikainen $P$, Alafuzoff I. The effect of prolonged fixation time on immunohistochemical staining of common neurodegenerative disease markers. J Neuropathol Exp Neurol 2010;69: 0-52.

18. Clarke JAL. Researches into the structure of the spinal cord. Phil Trans Roy Soc 1851;141:601-22.

19. Srinivasan M, Sedmak D, Jewell S. Effect of fixatives and tissue processing on the content and integrity of nucleic acids. Am J Pathol 2002;161:1961-71.

20. Cimmino A, Parisi G, Mastropasqua MG, Ricco R. A new technique for foetal brain fixation and extraction. Pathologica 2002;94:320-24.

21. Righini A, Parazzini C, Doneda C, Avagliano L, Arrigoni F, Rustico M, et al. Early formative stage of human focal cortical gyration anomalies: fetal MRI. Am J Roentgenol 2012;198:439-47.

22. Schulz G, Crooijmans HJ, Germann M, Scheffler K, Müller-Gerbl M, Müller B. Three-dimensional strain fields in human brain resulting from formalin fixation. J Neurosci Methods 2011;202:17-27.

23. Shatil AS, Uddin MN, Matsuda KM, Figley CR. Quantitative ex vivo MRI changes due to progressive formalin fixation in whole human brain specimens: Longitudinal characterization of diffusion, relaxometry, and myelin water fraction measurements at 3T. Front Med (Lausanne) 2018;5:31.

24. Schmued LC, Stowers CC, Scallet AC, $\mathrm{Xu}$ L. Fluoro-Jade $\mathrm{C}$ results in ultra high resolution and contrast labeling of degenerating neurons. Brain Res 2005;1035:24-31.

25. Sarkar S, Raymick J, Schmued LC. The use of recently developed histochemical markers for localizing neurotoxicant induced regional brain pathologies. Toxins (Basel) 2014;26:1453-70.

26. Sarubbo S, Basso G, Chioffi F, Cesnik E, Paradiso B, Grandi E, et al. Technical, anatomical, and functional study after removal of a symptomatic cavernous angioma located in deep Wernicke's territories with cortico-subcortical awake mapping. Case Rep Neurol Med 2013;2013: 835029.

27. Insausti N, Amaral DG. Hippocampal formation. In: G. Paxinos, editor. The human nervous system. Elsevier, London; 2004.

28. Marconi P, Zucchini S, Berto E, Bozac A, Paradiso B, Bregola G, et al. Effects of defective herpes simplex vectors expressing neurotrophic factors on the proliferation and differentiation of nervous cells in vivo. Gene Ther 2005;12:559-69.

29. Paradiso B, Marconi P, Zucchini S, Berto E, Binaschi A, Bozac A, et al. Localized delivery of fibroblast growth factor-2 and brain-derived neurotrophic factor reduces spontaneous seizures in an epilepsy model. P Natl Acad of Sci USA 2009;106:7191-6.

30. Roncon P, Zucchini S, Ferracin M, Marucci G, Giulioni M, Michelucci R, et al. Is autopsy tissue a valid control for epilepsy surgery tissue in microRNA studies? Epilepsia Open 2016;2:90-5.
31. Zucchini S, Marucci G, Paradiso B, Lanza G, Roncon P, Cifelli P, et al. Identification of miRNAs differentially expressed in human epilepsy with or without granule cell pathology. PLoS One 2014;9:e105521.

32. Zucchini S, Buzzi A, Bergamaschi M, Pietra C, Villetti G, Simonato M. Neuroprotective activity of CHF3381, a putative N-methyl-D-aspartate receptor antagonist. NeuroReport 2002;13:2071-4.

33. von Bezold A. [Untersuchungen uber die Vertheilung von Wasser, organischer Materia und anorganischen Verbindungen im Tierreiche]. Z Wiss Zool 1857;8:487. [Article in German].

34. Matsuda Y, Fujii T, Suzuki T, Yamahatsu K, Kawahara K, Teduka K, et al. Comparison of fixation methods for preservation of morphology, RNAs, and proteins from paraffin-embedded human cancer cell-implanted mouse models. J Histochem Cytochem 2011;59:68-75.

35. Zaglia T, Di Bona A, Chioato T, Basso C, Ausoni S, Mongillo M. Optimized protocol for immunostaining of experimental GFP-expressing and human hearts. Histochem Cell Biol 2016;146;407-19.

36. Thom M, Boldrini M, Bundock E, Sheppard MN, Devinsky O. The past, present and future challenges in epilepsy-related and sudden deaths and biobanking. Neuropathol Appl Neurobiol 2018;44:32-55.

37. Scagliotti V, Avagliano L, Gualtieri A, Graziola F, Doi P, Chalker J, et al. Histopathology and molecular characterisation of intrauterine-diagnosed congenital craniopharyngioma. Pituitary 2016;19:50-6.

38. Widdowson EM, Dickerson JW. The effect of growth and function on the chemical composition of soft tissues. Biochem J 1960;77:30-43. 